\title{
Análisis espacial de la convivencia ciudadana en el entorno geográfico del cerro Juan Rey parque Entrenubes de Bogotá D.C. ${ }^{1}$
}

\author{
Spatial analysis of citizen coexistence in the geographical \\ environment of the Juan Rey hill - parque Entrenubes of Bogotá D.C.
}

Edison Sarache Suárez ${ }^{2}$

\section{Resumen}

Este artículo muestra los resultados de una investigación aplicada de la geografía humana, mediante un análisis espacial de los factores que han afectado negativamente la convivencia ciudadana en el entorno geográfico del cerro Juan Rey, localizado en la periferia del suroriente de la ciudad de Bogotá. Se utilizó la evaluación multicriterio para establecer la relación entre la estratificación socioeconómica de los barrios con los mencionados factores, lo cual dio como resultado que no existe relación de causalidad espacial entre la estratificación socieconómica y las condiciones que afectan la convivencia ciudadana. Sin embargo, el modelo de análisis espacial permitió identificar aquellos barrios con alta conflictividad social que requieren de la intervención estatal y generó propuestas para el mejoramiento de las condiciones de convivencia ciudadana en este sector de la ciudad.

Palabras clave: análisis espacial, cerro Juan Rey, convivencia ciudadana, estratificación socioeconómica, evaluación multicriterio, geografía humana.

\footnotetext{
1 Este artículo es uno de los resultados de la investigación titulada Análisis Espacial de la Convivencia Ciudadana en el entorno geográfico Cerro Juan Rey Parque Entrenubes - Bogotá D.C.; enmarcada en la línea de investigación "Organización del Espacio" que pertenece al grupo de investigación Geografía y Ordenamiento Territorial -GEOT- de la Universidad Pedagógica y Tecnológica de Colombia, del programa académico de la Maestría en Geografía, como requisito para obtener el título de magister en Geografía.

2 Ingeniero Catastral y Geodesta, magister en Geografía, Programa de Estudios de Postgrado en Geografía EPG Convenio UPTC - IGAC. Colombia. edisarache@gmail.com
} 


\section{Abstract}

This paper shows the results of an applied human geography investigation through a spatial analysis of the factors that have negatively affected the peaceful coexistence in the geographical environment of the "Juan Rey" hill, located on the southeastern outskirts of Bogota. Multicriteria evaluation was used to establish the relationship between socioeconomic stratification of neighborhoods with the mentioned factors, which resulted in no relationship of spatial causality between socioeconomic stratification and conditions that affect citizen coexistence. However, the model of spatial analysis identified those neighborhoods with high social conflicts that require state intervention and generated proposals for improving the conditions of citizen coexistence in this part of the city.

Keywords: Spatial analysis, "Juan Rey" hill, citizen coexistence, socioeconomic stratification, multicriteria evaluation, Human Geography. 


\section{Introducción}

La Organización Mundial de la Salud -OMS- manifiesta que "La salud es un estado de completo bienestar físico, mental y social, y no solamente la ausencia de afecciones o enfermedades" (OMS, 1946). Reconoce que la violencia y la inseguridad son problemas de salud pública, puesto que han cobrado miles de vidas y han ocasionado discapacidad a muchas personas. Además, como lo menciona Acero (2002), los ciudadanos que viven en un ambiente de inseguridad y violencia son más susceptibles a ser víctimas de actos delictivos y a sentir miedo. Es precisamente esta patología social de la violencia y la delincuencia lo que interrumpe el equilibrio de la convivencia ciudadana, como condición de libertad de los ciudadanos de ejercer su libre derecho de coexistir en sociedad gozando de bienestar y tranquilidad sin sentirse víctimas de amenazas, miedos o violencia (Acero, 2002). Este problema ha sido tratado predominantemente por la criminología con base en estadísticas que incluyen la descripción de patrones que permiten explicar las conductas criminales y las características de las víctimas, y también ha atraído la atención de las ciencias sociales; pues, como lo explica Peña (1995), a medida que los criminólogos han tenido que considerar las cualidades espaciales de este fenómeno, ha sido necesario el apoyo de la ciencia geográfica para intervenir y dar expli- cación a estos problemas sociales que ocurren en un espacio geográfico.

Esta investigación se centra en el análisis espacial de aquellos problemas de violencia y delincuencia que han afectado la convivencia ciudadana en el entorno geográfico del cerro Juan Rey del Parque Ecológico Distrital Entrenubes ubicado en las unidades de planeamiento zonal (UPZ): 51 Los Libertadores de la localidad de San Cristóbal; 52 La Flora; 56 Danubio; 57 Gran Yomasa y 60 parque Entrenubes de la localidad de Usme; así como 55-Diana Turbay de la localidad Rafael Uribe Uribe. Esta zona, localizada en el suroriente de la ciudad de Bogotá D.C., se ha visto afectada por una alta intervención antrópica mediante actividad minera y urbanística que ha impactado sobre este sistema natural, además se caracteriza por poseer principalmente barrios de origen informal y que muestran condiciones de pobreza.

La problemática de la convivencia ciudadana en el área de estudio está relacionada con el comportamiento y actitudes de la sociedad que habita este territorio, el cual es entendido como un espacio que posee una configuración socioespacial producto de una dinámica de ocupación por diversas actividades económicas y oportunidades del mercado del suelo, dadas en su contexto histórico. La transformación de esta zona se caracterizó por la tran- 
sición de un área en donde predominaba la presencia de haciendas dedicadas a la producción de trigo y maíz, que después de 1950 sufrió cambios por causa de la explotación minera de canteras, y posteriormente fue urbanizada ante la necesidad suplir la demanda de los materiales de construcción que se requerían por el crecimiento de la ciudad de Bogotá y por la intensidad del desarrollo urbano que en su mayoría se ha determinado por el surgimiento de barrios de origen informal.

En esta investigación se identificaron como antecedentes algunos trabajos y disertaciones que han aportado al conocimiento de la geografía humana desde el punto de vista del análisis de la conflictividad urbana, los cuales han sido abordados a partir de la geografía del crimen, la geografía del miedo, la geografía de la violencia urbana y la geografía de la percepción, con un alto desarrollo en el ámbito anglosajón a la par con la ciencia criminológica que solo era tratada por la sociología y la psicología. Sin embargo, a finales de la década del 70 del siglo pasado surgieron estudios geográficos que incluyeron análisis espaciales de actos delictivos en los que se tienen en cuenta los factores de conflicto ocurridos en el espacio, así como la caracterización de la conducta de los agresores.

Por lo tanto, en esta línea del conocimiento geográfico, los resultados de esta investigación aportan metodología de análisis espacial de los conflictos generados por problemas en la convivencia, teniendo en cuenta la evaluación multicriterio, que permitió examinar las relaciones espaciales entre las manifestaciones que han alterado la convivencia ciudadana como delitos de alto impacto, conflictos que atentan contra la salud pública, conflictos por falta de tolerancia y presencia de población potencial generadora de actos violentos que fueron correlacionados con la estratificación socioeconómica.

\section{Descripción del área de estudio}

El área de estudio corresponde a los barrios del entorno geográfico del cerro Juan Rey que forma parte del Parque Ecológico Distrital de Montaña Entrenubes, ubicado al suroriente de la ciudad de Bogotá en las localidades 04 de San Cristóbal, 05 de Usme y 18 Rafael Uribe Uribe, que a su vez está contenida en seis unidades de planeamiento zonal (UPZ): 51 Los Libertadores, 52 La Flora, 55 Diana Turbay, 56 Danubio, 57 Gran Yomasa y 60 Parque Entrenubes, según fueron definidas por el POT (SDP, 2004). Ocupa una superficie de 1.380,90 ha, en un sistema montañoso que forma parte de la cuenca del río Tunjuelo y está atravesado por las subcuencas de las quebradas Chigüaza, La Hoya del Ramo, Santa Librada y Yomasa. Contiene 50 barrios de uso residencial en estratos 1 y 2 , y una población de 211.361 habi- 
tantes, según el censo de población del DANE del 2005.

Como ya se anotó, esta zona fue urbanizada principalmente de manera informal, y su población vive en condiciones socioeconómicas por debajo de la ciudad formal, lo cual se ve reflejado en la predominancia de estratos uno y dos (ver Figura 1).

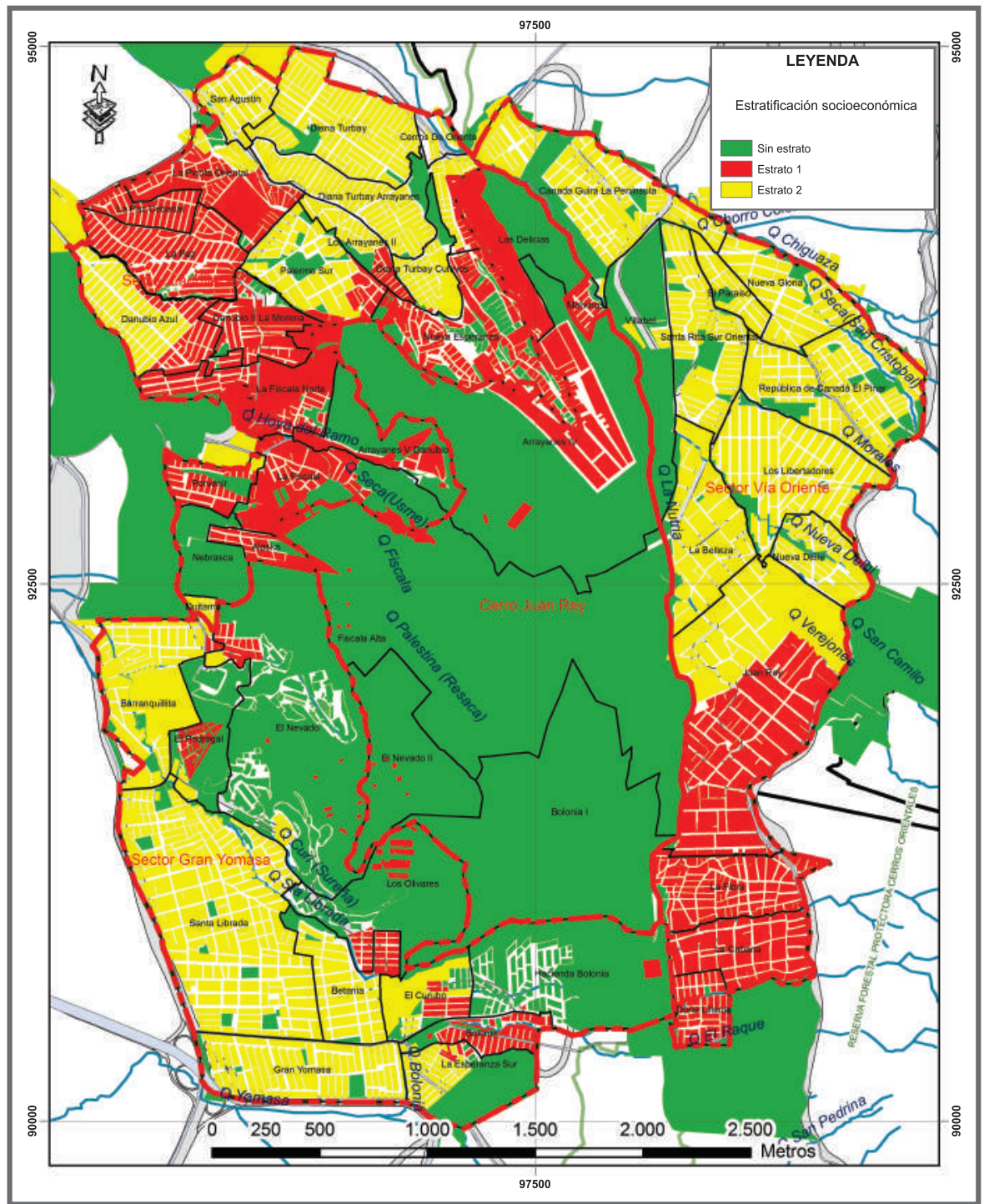

Fuente de Información cartográfica: SDP, 2004; UAECD, 2010

Fuente de datos de estratificación socioeconómica: Decreto distrital 176 de 2007 (SDP, 2007) Elaboración propia

Figura 1. Mapa de estratificación socioeconómica 


\section{Discusión conceptual}

En la relación que el ser humano ha tenido con el espacio, que por supuesto ha sido indispensable para su desarrollo, la geografía se ha convertido en una ciencia que ha permitido comprender la importancia de las dinámicas ocurridas por la interacción entre el hombre y el medio. Como lo menciona Santos (1997), al propio lugar se asocia una relación de flujos que recrean las condiciones ambientales y sociales y establecen acciones con un significado y valor para expresar una realidad geográfica. La discusión lleva a concluir que se requiere del análisis de la acción en el espacio, sin que el espacio sea el causante de los acontecimientos; de ahí la importancia de dar explicación a fenómenos y eventos que surgen como el producto de dicha interacción.

A fin de explicar las causas de la expresión geográfica de los seres humanos y la influencia de la naturaleza sobre estos, sobre su cultura y su historia (Gómez, 1983), y ante el predominio científico del positivismo, muchos geógrafos se centraron en una visión objetiva de los fenómenos humanos (Ortega, 2000). De modo que la nueva visión de la geografía humana propone que si el espacio es un área física ocupada por seres humanos, debe ser entendido teniendo en cuenta las dinámicas so- ciales. En otras palabras, el espacio no tiene significado por sí solo, puesto que es necesario comprender sus experiencias vividas, $\mathrm{y}$ al producto de este espacio socialmente construido, la geografía lo ha denominado "lugar".

Pero los lugares no son solo el producto de una interacción social ocurrida en un espacio, sino que también son el reflejo de nuestra identidad, que pueden ser transformados por hechos históricos, conflictos o intervenciones político-económicas, o como lo señala Sánchez (2012), que el espacio puede llegar a ser un factor conflictivo, dadas las distintas interpretaciones del mismo (Sánchez, 2012). De tal manera que para la comprensión de los conflictos sociales ocurridos en aquellos lugares ocupados por una comunidad, la ciencia geográfica se ha encargado de llevar a cabo trabajos que han aportado a la explicación de problemas similares que alteran la convivencia ciudadana, como la criminalidad, espacios del miedo y ocurrencia de delitos y conflictos, los cuales han sido abordados por la geografía del crimen, la geografía del miedo, la geografía de la violencia urbana y la geografía de la percepción.

También es importante definir la convivencia ciudadana, la cual, según el Centro de Estudio y Análisis en Convivencia y Seguridad Ciudadana 
-CEACSC- (2010), es "la condición y circunstancia de vivir con otros, lo que demanda una comunicación permanente, y expresión de sentidos y saberes, que al ponerse en escena, junto con procesos de conciliación, conducen a una relación armónica entre distintos individuos". Primero se convive cuando el ser humano nace y comienza a compartir su vida con otras personas, y cuando todas las condiciones de convivencia son favorables, le generan seguridad, y si aquella convivencia es vulnerada, la inseguridad ciudadana aparece; de esta manera se genera el conflicto o la inseguridad ciudadana. De hecho, el convivir en una ciudad implica el ejercicio de la ciudadanía, donde esta acción puede verse alterada por el conflicto, que es lo que hace que ocurra una desviación del orden de la convivencia (Red CONVIVAL, 2007)

En los años 70 del siglo pasado surgió la geografía de la delincuencia como apoyo a la criminología para la localización de actividades delictivas, y se desarrolló la escuela cartográfica de criminología, que se preocupó por analizar los comportamientos espaciales de la violencia urbana. La escuela ecológica de Chicago propuso la existencia de una interrelación entre la influencia del medio y el comportamiento criminal, logrando incluir la interpretación del crimen y la delincuencia como un indicador de calidad de vida o de progreso social, e incluso llegando a establecer una posible in- fluencia de la estratificación social y racial con el comportamiento de los delincuentes (Sanz, 2006).

La geografía del crimen, que tiene como antecedente la misma ciencia criminológica, se ubica en las ciencias sociales. A medida que la ciencia criminológica ha tenido que considerar las cualidades espaciales en sus estudios, ha recurrido a la ciencia geográfica para dar la explicación espacial a estos fenómenos. De ahí han surgido algunos estudios geográficos en los que se ha analizado el crimen y la delincuencia con la descripción de patrones espaciales que se han originado a partir de análisis estadísticos de las conductas criminales asociadas a los espacios geográficos y su posible incidencia sobre los mismos (Peña, 1995).

Las desigualdades y diversidades han sido fuentes de miedo en la ciudad, sin embargo la expresión del miedo que causa mayor relevancia es la originada por los actos delictivos. Al respecto, Francesco Indovina (citado por GGU \& AGE, 2005) se refiere a la ciudad como el "nicho ecológico de la espacie humana", y plantea como solución al miedo en la ciudad, el reconocimiento de la existencia del mismo y que hay elementos que configuran su código genético como la convivencia, el conflicto y el capital social, y que es necesaria más construcción de ciudadanía y espa- 
cios donde se maticen las desigualdades y se organicen las diferencias.

\section{Metodología}

La información primaria se obtuvo mediante la realización de cinco talleres, en los cuales participaron actores de la comunidad, como líderes y jóvenes, quienes con la coordinación de funcionarios públicos ubicaron sobre los mapas básicos, aquellos lugares en los cuales consideraban ellos que había presencia de consumo y expendio de sustancias psicoactivas y bebidas alcohólicas, y presencia de grupos potenciales generadores de actos violentos, como pandillas y habitantes de la calle percibidos por la comunidad durante el año 2009. Esta información fue representada por símbolos en los mapas trabajados en aquellos talleres.

La información secundaria se consiguió a partir de datos provenientes de las entidades públicas, especialmente del Centro de Estudios y Análisis en Convivencia y Seguridad Ciudadana (CEACSC) de la Secretaría Distrital de Gobierno (SDG) y el Sistema Unificado de Monitoreo de Impactos (SUMI) del Programa Sur de Convivencia (PSC) de la Secretaría Distrital del Hábitat (SDHT). El procesamiento de estos datos se hizo con la ayuda de los sistemas de información geográfica (SIG) con el software Arc-Gis 9.3, por medio del cual se realizó un análisis espacial con la metodología de evaluación multicriterio.

\section{La evaluación multicriterio como metodología de análisis espacial}

Para llevar a cabo el análisis espacial de los factores que han alterado la convivencia ciudadana, inicialmente se hizo una representación espacial de dicha problemática con la ayuda de los SIG, de la cual se obtuvieron mapas que muestran lo sucedido en aquel territorio durante el 2009. Con el análisis espacial se trataron problemas con múltiples componentes y variables, y se implementó la metodología de evaluación multicriterio (EMC).

La identificación de los indicadores y variables necesarios en el análisis espacial del problema se logró definiendo dos componentes generales: 1) las condiciones socioeconómicas; para tan fin se tuvo en cuenta la estratificación socioeconómica, y 2) las condiciones que afectan la convivencia ciudadana, como los delitos de alto impacto (homicidios, violencia sexual y hurtos), conflictos que atentan contra la salud pública (consumo y expendio de sustancias psicoactivas), conflictos por falta de tolerancia (violencia intrafamiliar y riñas) y presencia de población po- 
tencial generadora de actos violentos (pandilleros y habitantes de la calle). Y mediante la evaluación multicriterio (EMC) se definieron unas reglas de decisión que condujeron a la definición de unos pesos, como lo proponen Gómez \& Barredo (2005).

Para tal fin se elaboró una matriz que especifica la ponderación de cada una de estas variables, donde se asignaron pesos de criterio de acuerdo con las características de cada variable, dando el mayor peso a las condiciones de más alta conflictividad y menor paso a las menos impactantes. Cada indicador está conformado por un grupo de variables, donde a cada variable se le asigna un peso que está en una escala entre cero y uno, que indica el porcentaje de importancia de una variable respecto a la otra dentro de cada indicador (ver Tabla 1)

\subsection{Modelamiento espacial}

Una vez asignados los valores de la evaluación multicriterio, se procedió a calcular en las bases de datos de los objetos espaciales (polígonos de los barrios), mediante la ayuda del software ARC-GIS (V 9.3). Se realizaron las respectivas operaciones de los factores de cada variable para obtener así una base de datos con los resultados de la evaluación multicriterio, por medio de la aplicación de las fórmulas descritas en la matriz de formulación de indicadores y variables para la evaluación multicriterio (Tabla 1), lo que dio como resultado un valor para cada unidad espacial de análisis, que para efectos del diseño muestral, se clasificaron 50 unidades espaciales de análisis consideradas como barrios ${ }^{3}$.

El análisis espacial efectuado por medio del modelamiento matricial, ofrece varias posibilidades de la representación geográfica del problema. Así, se identificaron cuatro posibilidades de cruce de información entre las condiciones socioeconómicas y urbanísticas, y se tuvo en cuenta el índice de estratificación respecto a las condiciones que afectan la convivencia ciudadana, para ofrecer, por lo tanto, al planificador o a las entidades que intervienen en el territorio, criterios para focalizar proyectos de inversión hacia los barrios más críticos.

De acuerdo con este modelamiento matricial se esperan los siguientes resultados para cada indicador:

Índice de estratificación socioeconómica $(\mathrm{E})$ : se espera obtener con mayor valor, los barrios en los que predomina el estrato uno, que son aquellos que muestran mayores con-

3 Esta clasificación de barrios se llevó a cabo teniendo en cuenta la delimitación realizada por la Secretaría Distrital de Integración Social, en la que analizó el barrio considerando los aspectos culturales y el conocimiento de este por sus habitantes. 
Tabla 1. Matriz de formulación de indicadores y variables para la evaluación multicriterio

\begin{tabular}{|c|c|c|c|c|c|c|c|c|c|c|c|c|}
\hline & \multicolumn{3}{|c|}{ 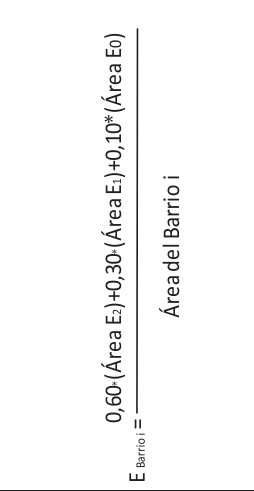 } & \multicolumn{3}{|c|}{ 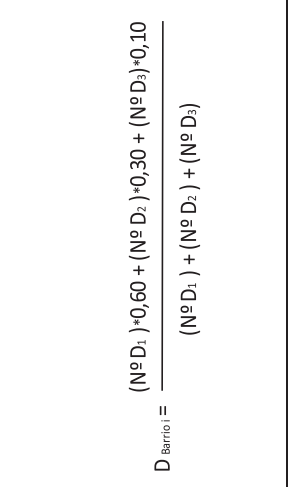 } & \multicolumn{2}{|c|}{ 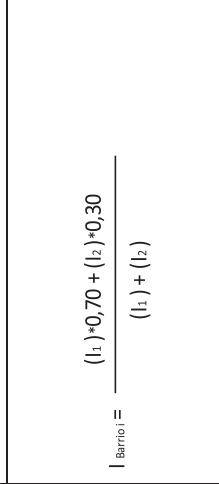 } & \multicolumn{2}{|c|}{ 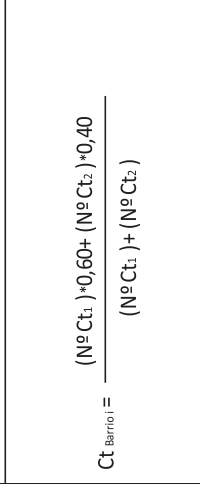 } & \multicolumn{2}{|c|}{ 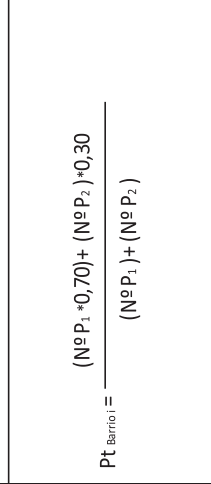 } \\
\hline & 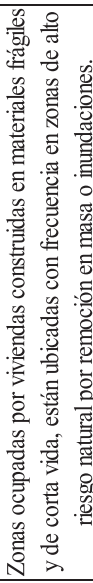 & 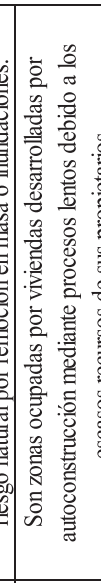 & 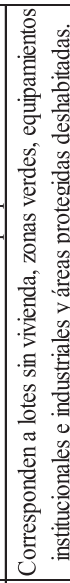 & 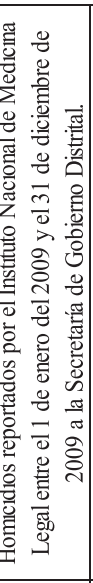 & 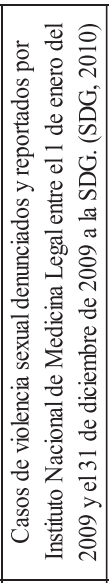 & 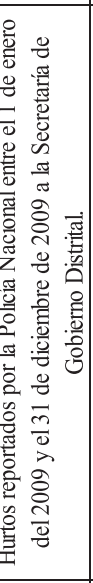 & 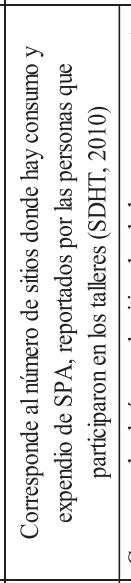 & 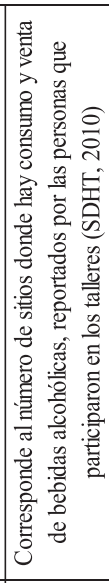 & 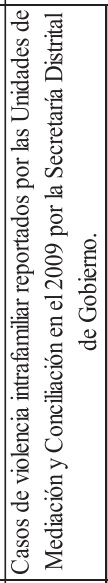 & 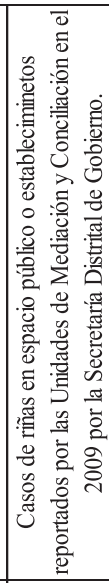 & 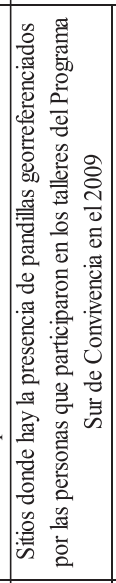 & 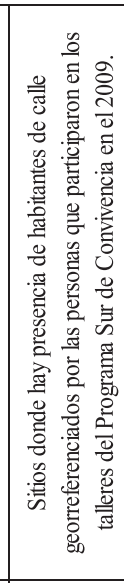 \\
\hline 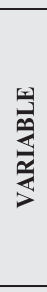 & 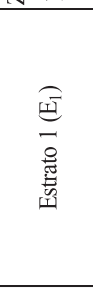 & 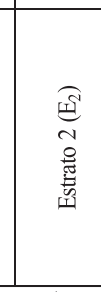 & 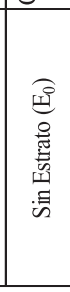 & 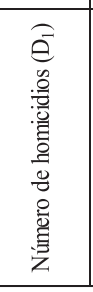 & 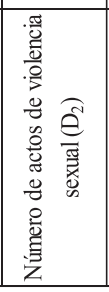 & 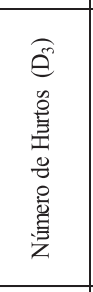 & 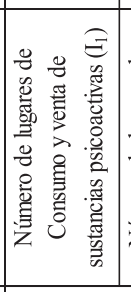 & 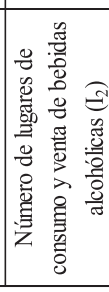 & 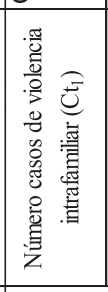 & 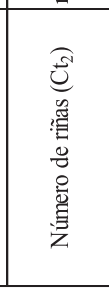 & 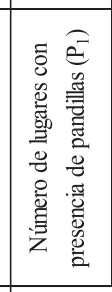 & 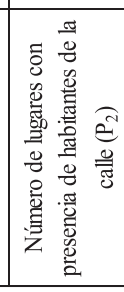 \\
\hline 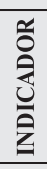 & & 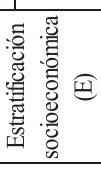 & & & 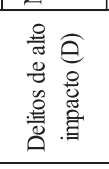 & & 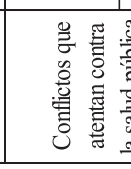 & 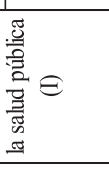 & 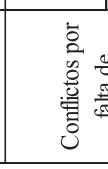 & & 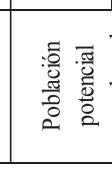 & 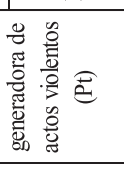 \\
\hline 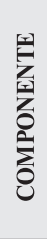 & & 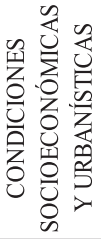 & & & & & & 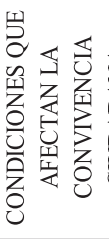 & & & & \\
\hline
\end{tabular}

Fuente: elaboración propia 
diciones de pobreza, precariedad en sus edificaciones y vías en mal estado, según la metodología de estratificación socioeconómica implementada en la ciudad de Bogotá por la Secretaría Distrital de Planeación (SDP, 2005).

Índice de delitos de alto impacto (D): se espera obtener a mayor intensidad de delitos mayor valor en el índice, es decir, que los barrios donde ocurre la mayor cantidad de homicidios, violaciones sexuales y hurtos, serán aquellos de mayor valor.

Índice de conflictos que atentan contra la salud pública (I): con este índice se espera obtener con mayor valor aquellos barrios que poseen la mayor presencia de sitios donde predomina el consumo y venta de sustancias psicoactivas (drogas ilegales y bebidas alcohólicas).

Índice de conflictos por falta de tolerancia $(\mathrm{Ct})$ : con este índice se espera obtener con mayor valor aquellos barrios en donde ocurrieron con mayor frecuencia casos de violencia intrafamiliar y riñas.

Índice de población potencial generadora de actos violentos $(\mathrm{Pt})$ : con este índice se espera obtener mayor valor para aquellos barrios en los que hay mayor presencia de pandillas y habitantes de calle.

El mapa correspondiente a la Figura 3 muestra los resultados de los in- dicadores de condiciones de convivencia mediante diagrama de columnas, donde la altura de cada columna representa la intensidad del delito o conflicto, es decir, que a mayor conflicto mayor altura o viceversa. Lo que permite comparar visualmente los indicadores de las condiciones que afectan la convivencia ciudadana respecto al índice de estratificación para cada barrio, que es representado por medio de coropletas asociadas al polígono de cada barrio, mostrando una variación en el color, donde cada color significa el grado de estratificación socioeconómica. Así, el color rosado muestra las condiciones que obtuvieron mayor puntaje en la evaluación multicriterio, al que se relacionan las condiciones socioeconómicas más precarias; el amarillo indica el rango medio, y el verde señala los barrios que obtuvieron la puntuación más baja, que corresponde a aquellos que cuentan con las mejores condiciones de estratificación.

\subsection{Asignación de pesos y valores para la evaluación multicriterio}

Aquí se cita un aspecto muy relevante que es la síntesis del componente de las condiciones que afectan la convivencia ciudadana respecto a las condiciones urbanísticas de los barrios, reflejada en la estratificación socioeconómica para cada uno de los 
sectores; de modo que el objetivo que es pertinente analizar, consiste en identificar cuáles son los barrios con más alta conflictividad social respecto a la estratificación socioeconómica, y que el resultado sirva para formular propuestas de mejoramiento en aquellos barrios.

Hay que aclarar que la asignación de estos pesos puede variar de acuerdo con el criterio del planificador, sin embargo sirve de base para el modelamiento de un escenario de evaluación propuesto, como lo señalan metodológicamente Gómez y Barredo en la evaluación multicriterio y multiobjetivo en el entorno de los sistemas de información geográfica (Gómez \& Barredo, 2005, p. 53). Para tal fin se definieron los indicadores, variables y criterios en la evaluación multicriterio de conformidad con la matriz de formulación de las variables ilustradas en la Tabla 1.

\section{Resultados y discusión}

El mapa correspondiente a la Figura

3 sintetiza los resultados del procesamiento estadístico de acuerdo con la evaluación multicriterio, y muestra como base las condiciones de estratificación socioeconómica representadas por coropletas. Mediante diagrama de columnas se expresan las condiciones que han afectado la convivencia ciudadana en el área de estudio, donde la altura describe el valor del índice calculado con arreglo a las fórmulas relacionadas en la Tabla 1.

Para comprobar si es posible que exista alguna relación de causalidad entre el grado de estratificación socioeconómica y las condiciones que afectan la convivencia ciudadana, se calcularon los coeficientes de determinación de la correlación de Pearson, teniendo como variable independiente las condiciones de estratificación (E) y como variables dependientes las condiciones que afectan la convivencia ciudadana, que corresponden a los siguientes indicadores: 1) delitos de alto impacto (D), 2) conflictos que atentan contra la salud pública (I), 3) conflictos por falta de tolerancia $(\mathrm{Ct})$ y 4) población potencial generadora de actos violentos $(\mathrm{Pt})$. Se consiguieron los siguientes resultados:

Tabla 2. Coeficientes de determinación de la correlación de Pearson

\begin{tabular}{|c|c|c|c|c|c|}
\hline & $E$ & $D$ & $I$ & $C t$ & $P t$ \\
\hline$E$ & 100,00 & 11,47 & 10,54 & 14,72 & 19,72 \\
\hline
\end{tabular}

Fuente: elaboración propia 
Esta tabla de coeficientes de determinación de la correlación de Pearson sirve para mostrar el porcentaje de correlación de un indicador respecto al otro (Nieves \& Domínguez). A partir de este resultado se deducen las condiciones de estratificación socioeconómica (E) referidas al grado de intensidad de cada uno de los indicadores de condiciones que afectan la convivencia ciudadana $(\mathrm{D}, \mathrm{I}, \mathrm{Ct}$ y Pt). Para este caso se encontraron porcentajes de correlación muy débiles, los cuales mostraron que la correlación que posee el más alto valor es entre las condiciones de estratificación (E) y el índice de población potencial de actos violentos $(\mathrm{Pt})$; lo que dio como resultado un 19,72\% de correlación, como lo muestra la Figura 2.

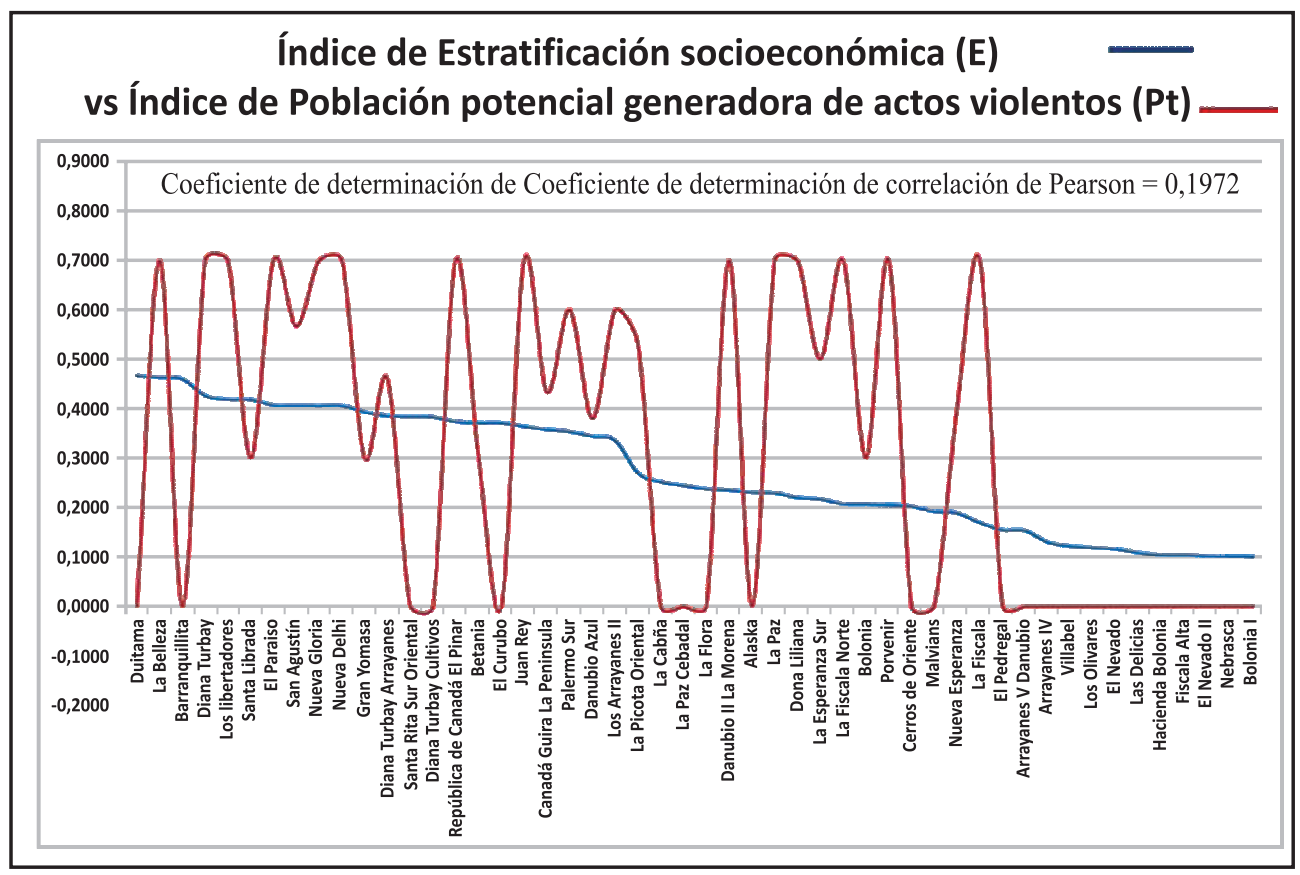

Figura 2. Correlación entre el Índice de tipo de estratificación socioeconómica vs Índice de población potencial generadora de actos violentos.

Fuente: elaboración propia 


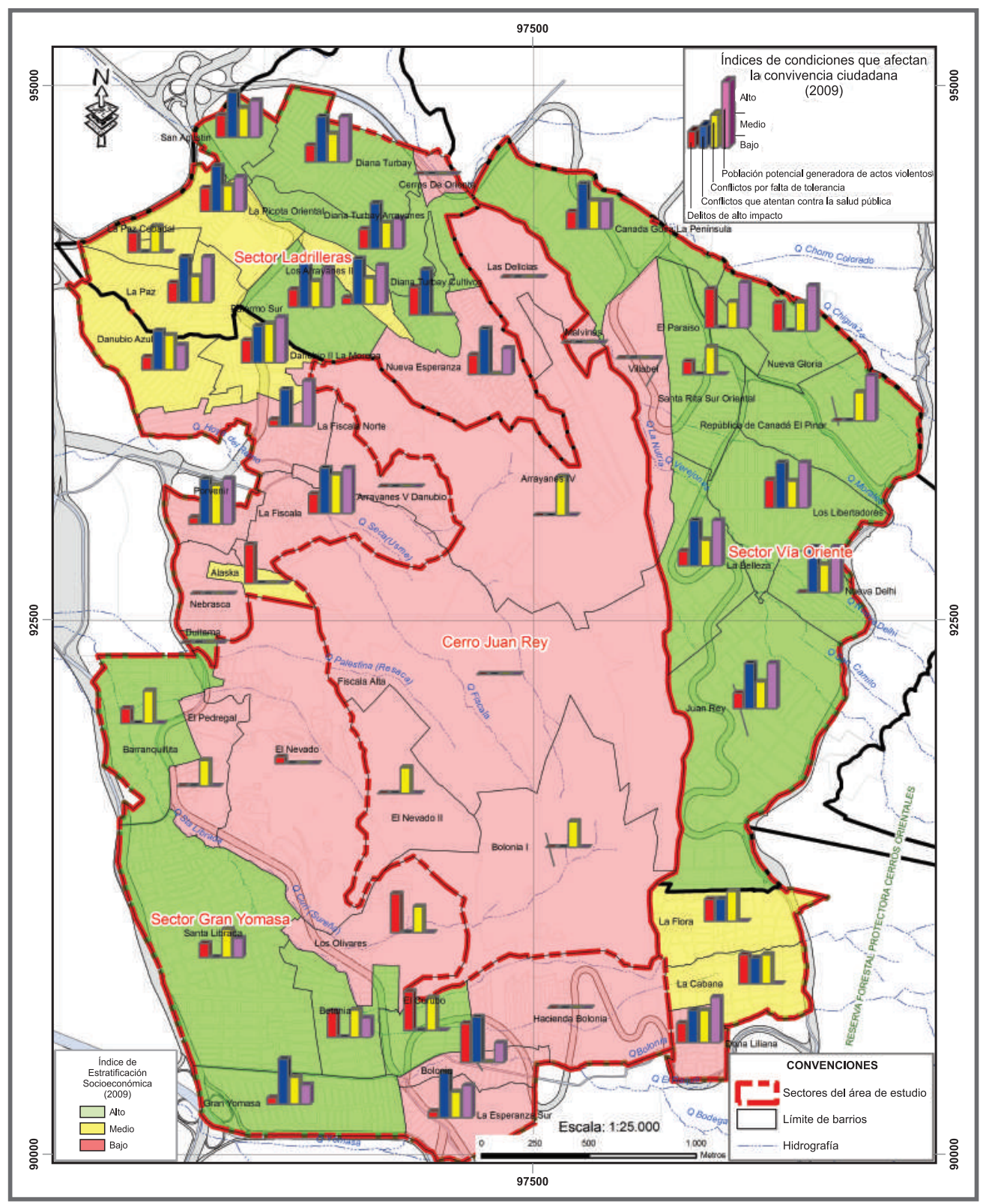

Fuente de Información cartográfica: SDP, 2004; UAECD, 2010

Fuente de datos de índice de la estratificación socioeconómica: Decreto distrital 176 de 2007 (SDP, 2007) Fuente de datos de condiciones que afectan la convivencia: SDG, 2009; SDHT, 2009 Cálculos y Resultados de Evaluación Multicriterio: Elaboración propia

Figura 3. Mapa de condiciones que afectan la convivencia ciudadana respecto a la estratificación socioeconómica 


\section{Propuestas}

Para mejorar las condiciones de estratificación se recomienda la ejecución de obras encaminadas a arreglar tanto las condiciones individuales de las viviendas como las del entorno; por ejemplo, la reparación de vías, espacios públicos, alumbrado público; la construcción de áreas recreacionales y deportivas; el mantenimiento de zonas verdes y la optimización de las redes de servicios públicos.

Con base en la tabla de coeficientes de determinación de la correlación de Pearson (Tabla 2), en la cual el índice de condiciones de estratificación (E) versus el índice de población potencial de actos violentos (Pt), da un valor igual a $19,72 \%$, la intervención sugerida apunta a mejorar las condiciones socioeconómicas de la población potencial causante de actos delictivos, para lo cual es necesario establecer mecanismos de generación de oportunidades y empleo para esta población, focalizándose especialmente en los barrios La Belleza, Diana Turbay, Los Libertadores y El Paraíso, según el resultado de la Figura 2.

Para tal fin se propone la implementación de los siguientes programas que conforman la oferta institucional:

El Programa de Mejoramiento Integral de Barrios, dirigido a mejorar las condiciones de habitabilidad, el estado de las vías locales, el acceso a los servicios públicos y la legalización de los barrios, y cuyas entidades responsables son la Secretaría Distrital del Hábitat, la Caja de Vivienda Popular y las empresas de servicios públicos.

Para mejorar las condiciones de pobreza se propone la participación de la Secretaría Distrital de Desarrollo Económico y el IPES, a fin de ayudar a la promoción de proyectos productivos y generación de oportunidades laborales.

Otro frente de intervención que se propone es el programa "Salud a su casa" de la Secretaría Distrital de Salud, que se encarga de evaluar las condiciones de vulnerabilidad de las familias más pobres con el fin de brindarles ayudas para el mejoramiento de las condiciones de habitabilidad de las viviendas más vulnerables.

\section{Conclusiones}

Con el examen espacial de los factores que han afectado negativamente la convivencia ciudadana en el área del estudio, mediante la metodología de evaluación multicriterio y el análisis de correlación de Pearson, se pudo comprobar que no existe correlación entre las condiciones de estratificación respecto a las condiciones 
que alteran la convivencia pacífica. De aquí se deduce que la pobreza o la precariedad de las viviendas no es determinante en la conflictividad de los barrios, aunque para poder asegurar ello es necesario efectuar más observaciones en la ciudad $\mathrm{u}$ otras ciudades.

Mediante los sistemas de información geográfica se pueden representar espacialmente problemáticas sociales, como aquellos factores que alteran la convivencia pacífica de algún lugar, lo cual le permite al planificador tomar decisiones de mejoramiento en el área estudiada, y con la ayuda de la evaluación multicriterio puede obtener valores de cada uno de los indicadores formulados, para después calcularles la correlación de Pearson y así establecer criterios para privilegiar programas $\mathrm{y}$ proyectos encaminados a mejorar las condiciones conflictivas del área de estudio.

\section{Referencias}

Acero, H. (2002). Ciudad y políticas públicas de seguridad y convivencia. Cómo se logró reducir la violencia y la delincuencia en Bogotá, Colombia 1995-2001. Bogotá: Alcaldía Mayor de Bogotá D.C., Secretaría de Gobierno.

Centro de Estudios y Análisis en Convivencia y Seguridad Ciudadana -CEACSC-. (2010). Guía del buen gobierno para la seguridad ciudadana. Bogotá: Secretaría Distrital de Gobierno, CEACSC.

Díaz, C. A. (2009). Algunas características del proceso de urbanización de Bogotá desde 1950 hasta finales del siglo xx. Una mirada desde la marginalidad social urbana. Recuperado de http://carlosdiazmosquera.blogspot.com/2009/04/proceso-deurbanizacion.html

Departamento Nacional de Planeación -DNP-. (2004). CONPES 3326 del 2004: garantía de la Nación a Bogotá D.C. para contratar un empréstito externo con el gobierno alemán por medio del Kreditanstalt für wiederaufbau ( $k f w)$ por $€ 33,55$ millones, o su equivalente en otras monedas, para la financiación del Programa Sur de Convivencia. Bogotá: DNP.

Grupo de Geografía Urbana -GGU- \& Asociación de Geógrafos Españoles -AGE- (2005). La ciudad y el miedo. Coloquio de geografía urbana. Girona, España: GGU, AGE, Universitat de Girona.

Gómez, A. L. (1983, nov.). La geografía humana: ¿de la ciencia de los lugares a ciencia social? Cuadernos Críticos de Geografía Humana - Geocrítica, Año VIII (Número 48), Recuperado de http://www.ub.edu/geocrit/geo48.htm

Gómez, M. \& Barredo, J. I. (2005). Sistemas de Información geográfica y evaluación multicriterio. México D.F.: Alfaomega. 
Nieves, A. \& Domínguez, F. (2009). Probabilidad y estadística para ingeniería. Ed. Mcgraw Hill. Recuperado de http://www.mcgraw-hill-educacion.com/pye01e/ cap13/13analisis_de_correlacion_y_regresion.pdf

Organización Mundial de la Salud -OMS- (1946). Constitución de la Organización Mundial de la Salud. New York: OMS. Recuperado de http://apps.who.int/gb/bd/PDF/bd47/ $\mathrm{SP} /$ constitucion-sp.pdf

Ortega, José (2000): Los horizontes de la geografía. Barcelona: Ed. Ariel Geografía.

Peña, L. A. (1995). Ciudad Bolívar y la geografía del crimen. Tesis para optar al título de magíster en Geografía. Programa de Estudios de Posgrado en Geografía -EPG- de la Universidad Pedagógica y Tecnológica de Colombia - UPTC, Bogotá: UPTC-IGAC.

Red CONVIVAL. (2007). Políticas urbanas y convivencia en ciudades de América Latina: hacia una gestión integral de la ciudad. Bogotá: UNESCO.

Sánchez, L. (2012). Geografía humana: conceptos básicos y aplicaciones. Bogotá: Universidad de los Andes, Facultad de Ciencias Sociales, Departamento de Historia, Centro de Estudios Socioculturales e Internacionales -CESO-.

Santos, M. (1997). La naturaleza del espacio. Técnica y tiempo, razón y emoción. Barcelona: Ariel.

Secretaría Distrital del Hábitat -SDHT-. (2011). Convenio de asociación no. 082 de 2011 celebrado entre la Secretaría Distrital del Hábitat y el Centro de Estudios de la Construcción y el Desarrollo Urbano y Regional, CENAC. Fase 5 el proceso de la ciudad informal. Bogotá: SDHT.

Secretaría Distrital de Planeación -SDP- (2004). Decreto 190 de 2004: por medio del cual se compilan las disposiciones contenidas en los decretos distritales 629 de 2000 y 469 de 2003. Bogotá: SDP.

Secretaría Distrital de Planeación -SDP-. (2005). La estratificación de Bogotá D.C. y estudios relacionados 1983-2004. Bogotá: SDP.

Secretaría Distrital de Planeación -SDP- (2011). 21 Monografías de las localidades, Distrito Capital 2011, Diagnóstico de los aspectos físicos, demográficos y socioeconómicos año 2011, Localidad 4 San Cristóbal. Recuperado de http://www.sdp.gov.co/ portal/page/portal/PortalSDP/Informaci\%F3nTomaDecisiones/Estadisticas/ Documentos:An\%E1lisis 
Recepción: 01 de octubre de 2013

Evaluación: 11 de noviembre de 2013

Aprobación: 16 de diciembre de 2013 